\title{
miRNAs and SAMHD1 regulation in vitro and in a model of HIV CNS disease
}

\author{
Kenneth W. Witwer ${ }^{*}$ D, Erin L. Buchanan, Stephanie L. Myers and Melissa A. McAlexander
}

\begin{abstract}
Pilakka-Kanthikeel et al. recently reported higher levels of the retroviral restriction factor sterile alpha motif and histidine/aspartic acid domain-containing protein 1 (SAMHD1) in astrocytes than in microglia, suggesting that SAMHD1 levels might explain in part the relatively refractory nature of astrocytes to retroviral replication. These findings are consistent with our studies of simian and human immunodeficiency virus infection of astrocytes and macrophages. Similarly, a role for two host microRNAs in post-transcriptional regulation of SAMHD1 agrees with our in vitro results and those of others. However, data from an animal model of HIV neurologic disorders may not be consistent with robust miRNA-mediated regulation of SAMHD1 in vivo.
\end{abstract}

\section{Letter to the editor}

We were pleased to read the recent article of PilakkaKanthikeel and colleagues, reporting higher levels of the retroviral restriction factor sterile alpha motif and histidine/ aspartic acid domain-containing protein 1 (SAMHD1) in astrocytes than in microglia [1]. The data suggest that SAMHD1 levels might explain in part the relatively refractory nature of astrocytes to retroviral replication. These findings are consistent with our studies of simian and human immunodeficiency virus (SIV and HIV) infection of astrocytes and macrophages ([2], "Regulation of the SAMHD1 transcript during retroviral infection of the central nervous system," submitted); see also the different results on SAMHD1 in elite suppressor populations [3-5]. Similarly, a role for two host microRNAs (miRNAs) in post-transcriptional regulation of SAMHD1 agrees with our results [2] and Swaminathan et al. [6].

At the same time, we are somewhat uncertain about the extent to which these miRNAs regulate SAMHD1 in vivo. Pilakka-Kanthikeel et al. transfected $50 \mathrm{nM}$ miR155 and miR-181 mimic or inhibitor and observed effects on SAMHD1 levels in cultured astrocytes [1]. In our hands, $10 \mathrm{nM}$ miR-155 and miR-181-as well as several others including miR-34a and miR-150-reduced the expression of luciferase reporters fused to the SAMHD1 3' untranslated region (3' UTR), supporting

\footnotetext{
* Correspondence: kwitwer1@jhmi.edu

Department of Molecular and Comparative Pathobiology, The Johns Hopkins
University School of Medicine, 733 North Broadway, BRB Suite 831, Baltimore,

Department of Molecular and Comparative Pathobiology, The Johns Hopkins MD 21025, USA
}

direct regulation (Fig. 1a). However, the stoichiometries of these "all or nothing" assays are highly artificial, with exposures amounting to millions or tens of millions of oligonucleotides per cell. Additionally, and as reported previously by others [7], we observed significant cell death with miR-181a transfection, a potential explanation for the particularly large reduction in this condition. The authors did not report viability in their populations, but we wonder if this could explain some of the observed effects.

Seeking to determine whether there is a tissue-level, in vivo correlation between SAMHD1 and miRNAs found regulate the transcript in vitro, we measured SAMHD1 of HIV disease [8], including uninfected controls. tly downregulated $(p<0.001)$. This regulation in irections could be interpreted as consistent with 作 any of the following groups: acute phase infection (Fig. 1b), progression to disease (Fig. 1c), or all samples, including uninfected controls (Fig. 1d). There was also no correlation of SAMHD1 with levels of miR-155 or miR-34a, which in fact tended to increase during infection (data not shown). These results recall a previous finding in which the RNA binding protein ZFP36 (tristetraprolin) could be suppressed by miRNAs in vitro but showed little evidence of negative correlation with the same miRNAs in vivo [9]. 
A

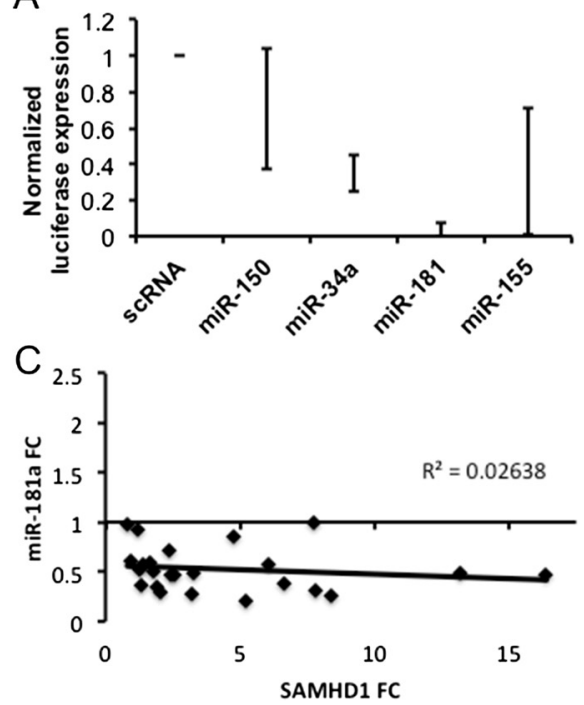

$\mathrm{B}$

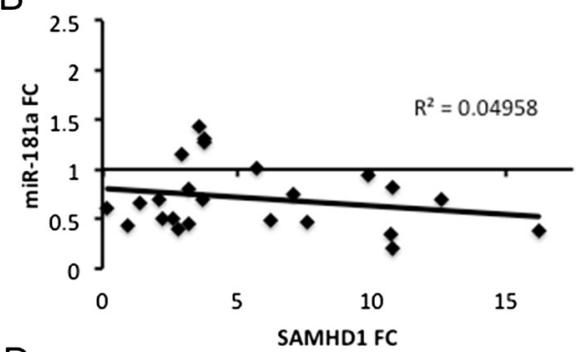

D

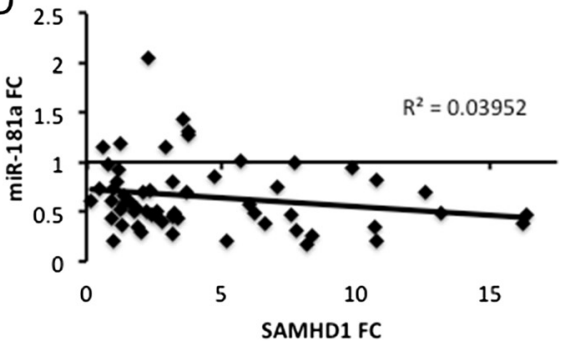

Fig. 1 SAMHD1 and miRNAs in vitro and in vivo. a miRNAs suppress SAMHD1-linked reporter assay. 293T cells in 96-well plates were transfected with $10 \mathrm{nM}$ scrambled RNA control (scRNA) or $10 \mathrm{nM}$ miRNA mimics (Life Technologies), along with 50 ng luciferase reporter vector containing the human SAMHD1 3' UTR (SwitchGear Genomics) or empty vector. After 48-72 h, luciferase activity was measured using a Fluoroskan Ascent fluorometer (Thermo Scientific). b-d Normalized fold change of thalamic SAMHD1 and miR-181a expression compared with the average of uninfected controls. Total RNA was extracted from the archived thalamus samples from an SIV model of HIV disease. SAMHD1 levels were normalized to the average of GAPDH and actin beta expression using data collected using hydrolysis probe qPCR assays (Life Technologies). Expression of miR-181a (shown) and other miRNAs (including miR-34a and miR-155, not shown) were determined by stem-loop reverse transcription/qPCR assays (Life Technologies) and normalized to U6 snRNA. Correlations of miR-181a and SAMHD1 expression are shown for $\mathbf{b}$ subjects during acute phase infection (4-14 days post infection), c subjects during progression to disease (21-84 days p.i.), and $\mathbf{d}$ for all samples, including uninfected controls. Each point represents one subject. No correlations were significant $(p<0.05)$ or trended towards significance $(p<0.1)$

While our findings temper our initial enthusiasm about miRNA-mediated control of SAMHD1, we are not prepared to dismiss it entirely. Although the astrocyte is the most abundant cell type in the brain and appears to support higher levels of SAMHD1 expression than, e.g., macrophages ["Regulation of the SAMHD1 transcript during retroviral infection of the central nervous system," submitted] and microglia [1], the tissue-level profiling we performed may mask changes of miRNA or SAMHD1 expression in specific cells types or even the absence of certain miRNAs in disease-related cells [10]. Similarly, focusing on single miRNA-to-target interactions ignores the regulatory "whole picture," since a given miRNA-to-target interaction is governed not only by the abundance of each partner but also by the abundance of other partners [11]. Finally, assuming adequate delivery methods, therapeutic antisense manipulation of anti-SAMHD1 miRNAs (for example, to help prevent cell-to-cell transmission during eradication therapy) could be performed at high concentrations not dissimilar to the in vitro manipulations that we and the authors performed. There may will be more to learn about miRNAs and SAMHD1 in the CNS.

\section{Abbreviations}

3' UTR: 3' untranslated region; CNS: central nervous system; HIV: human immunodeficiency virus; miRNA: microRNA; SAMHD1: SAM-domain and HD-domain containing protein 1.

\section{Competing interests}

The authors declare that they have no competing interests.

\section{Authors' contributions}

KWW conceived of, designed, and managed the study. ELB, MAM, SLM, and KWW performed experiments. ELB and KWW analyzed results. KWW wrote the manuscript. All authors read and approved the final manuscript.

\section{Acknowledgements}

This work was supported in part by NIH grant R21 Al102659 (KWW), NIMH pilot grant P30 MH075673 (to KWW), and by the National Center for Research Resources and the Office of Research Infrastructure Programs (ORIP) and the National Institutes of Health through grant number P40 OD013117.

Received: 5 August 2015 Accepted: 17 August 2015

Published online: 04 September 2015

\section{References}

1. Pilakka-Kanthikeel S, Raymond A, Atluri VS, Sagar V, Saxena SK, Diaz P, et al. Sterile alpha motif and histidine/aspartic acid domain-containing protein 1 (SAMHD1)-facilitated HIV restriction in astrocytes is regulated by miRNA-181a. J Neuroinflammation. 2015;12(1):66.

2. Buchanan EL, Espinoza DA, Sisk JM, Moyer A, Clements JE, Witwer KW. Expression and regulation of host restriction factor SAMHD1 during lentiviral infection of astrocytes and macrophages. In: Conference on Retroviruses and Opportunistic Infections. Atlanta, GA, USA; CROI, LLC, Alexandria, 2013

3. Riveira-Munoz E, Ruiz A, Pauls E, Permanyer M, Badia R, Mothe B, et al. Increased expression of SAMHD1 in a subset of HIV-1 elite controllers. J Antimicrob Chemother. 2014;69:3057-60.

4. Abdel-Mohsen M, Raposo RA, Deng X, Li M, Liegler T, Sinclair E, et al. Expression profile of host restriction factors in HIV-1 elite controllers. Retrovirology. 2013;10:106. 
5. Buchanan EL, McAlexander MA, Witwer KW. SAMHD1 expression in blood cells of HIV-1 elite suppressors and viraemic progressors. J Antimicrob Chemother. 2014;70:954-6.

6. Swaminathan G, Navas-Martin S, Martin-Garcia J. MicroRNAs and HIV-1 infection: antiviral activities and beyond. J Mol Biol. 2014;426(6):1178-97.

7. Ouyang YB, Lu Y, Yue $S, X u L J$, Xiong XX, White RE, et al. miR-181 regulates GRP78 and influences outcome from cerebral ischemia in vitro and in vivo. Neurobiol Dis. 2012:45(1):555-63.

8. Beck SE, Queen SE, Witwer KW, Metcalf Pate KA, Mangus LM, Gama L, et al. Paving the path to HIV neurotherapy: predicting SIV CNS disease. Eur J Pharmacol. 2015;759:303-12.

9. Liu J, Sisk JM, Gama L, Clements JE, Witwer KW. Tristetraprolin expression and microRNA-mediated regulation during simian immunodeficiency virus infection of the central nervous system. Mol Brain. 2013;6(1):40.

10. Kent OA, McCall MN, Cornish TC, Halushka MK. Lessons from miR-143/145: the importance of cell-type localization of miRNAs. Nucleic Acids Res. 2014:42(12):7528-38

11. Seitz H. Redefining microRNA targets. Curr Biol. 2009;19(10):870-3.

\section{Submit your next manuscript to BioMed Central and take full advantage of:}

- Convenient online submission

- Thorough peer review

- No space constraints or color figure charges

- Immediate publication on acceptance

- Inclusion in PubMed, CAS, Scopus and Google Scholar

- Research which is freely available for redistribution 